\title{
The mitochondrial anti-apoptotic dependencies of hematologic malignancies: from disease biology to advances in precision medicine
}

\author{
Isacco Ferrarini, Antonella Rigo and Carlo Visco \\ Department of Medicine, Section of Hematology, University of Verona, Verona, Italy
}

Haematologica 2022

Volume 107(4):790-802

\section{Correspondence:}

ISACCO FERRARINI

isacco.ferrarini@univr.it

CARLO VISCO

carlo.visco@univr.it

Received: October 18, 2021.

Accepted: January 7, 2022.

Prepublished: January 20, 2022.

https://doi.org/10.3324/haematol.2021.280201

(C)2022 Ferrata Storti Foundation

Material published in Haematologica is covered by copyright. All rights are reserved to the Ferrata Storti Foundation. Use of published material is allowed under the following terms and conditions:

https://creativecommons.org/licenses/by-nc/4.0/legalcode. Copies of published material are allowed for personal or internal use. Sharing published material for non-commercial purposes is subject to the following conditions:

https://creativecommons.org/licenses/by-nc/4.0/legalcode, sect. 3. Reproducing and sharing published material for commercial purposes is not allowed without permission in writing from the publisher.

\section{ABSTRACT}

1 itochondria are critical organelles in the regulation of intrinsic apoptosis. As a general feature of blood cancers, different antiouter mitochondrial membrane to sequester variable amounts of proapoptotic activators, and hence protect cancer cells from death induction. However, the impact of distinct anti-apoptotic members on apoptosis prevention, a concept termed anti-apoptotic dependence, differs remarkably across disease entities. Over the last two decades, several genetic and functional methodologies have been established to uncover the anti-apoptotic dependencies of the majority of blood cancers, inspiring the development of a new class of small molecules called $\mathrm{BH} 3$ mimetics. In this review, we highlight the rationale of targeting mitochondrial apoptosis in hematology, and provide a comprehensive map of the anti-apoptotic dependencies that are currently guiding novel therapeutic strategies. Cell-extrinsic and -intrinsic mechanisms conferring resistance to $\mathrm{BH} 3$ mimetics are also examined, with insights on potential strategies to overcome them. Finally, we discuss how the field of mitochondrial apoptosis might be complemented with other dimensions of precision medicine for more successful treatment of 'highly complex' hematologic malignancies.

\section{Introduction}

Prevention of programmed cell death is a hallmark of cancer cells and efforts to re-establish pro-death pathways have been the mainstay of research in the field of anti-cancer therapeutics. ${ }^{1}$ Among modalities of programmed cell death, apoptosis is the best characterized in terms of triggering stimuli, sequencing of biochemical events, intracellular organelles involved, and morphological changes. ${ }^{2}$ Two interconnected forms of apoptosis have been described: the extrinsic and the intrinsic (i.e., mitochondrial) pathways. The former is triggered on the cell surface by the engagement of death receptors, such as tumor necrosis factor receptor and tumor necrosis factor-related apoptosis-inducing ligand receptor, and proceeds through caspase- $8 / 10$ activation and BID cleavage. ${ }^{3}$ The latter is induced by oncogenic signaling, nutrient deprivation, genotoxic drugs and other cellular stressors, and is regulated at the level of the outer mitochondrial membrane by pro- and anti-apoptotic BCL-2 family members. ${ }^{4}$

\section{Overview of the BCL-2 family}

The BCL-2 family members are distinguished into three main categories: antiapoptotic, pro-apoptotic BH3-only, and pro-apoptotic effectors ${ }^{4}$ (Figure 1). BCL-2, MCL-1, BCL-w, BCL-B, BCL-xL, and BFL-1 are the main pro-survival relatives and contain all four $\mathrm{BH}$ domains., $\mathrm{AID}$, BIM, PUMA, NOXA, BAD, HRK, and BMF are the pro-apoptotic $\mathrm{BH} 3$-only proteins, and only share the $\mathrm{BH} 3$ domain with the rest of the family. Among these, BID and BIM can directly activate the pro-apoptotic effectors, while the others act as sensitizers by antagonizing the pro-survival 
members. ${ }^{6} \mathrm{BAX}, \mathrm{BAK}$, and $\mathrm{BOK}$ contain three out of four $\mathrm{BH}$ domains and represent the pro-apoptotic effector proteins that form homodimers and heterodimers through the outer mitochondrial membrane. ' Overall, the mitochondrial cascade of apoptosis starts when a death stimulus triggers the translocation of $\mathrm{BH} 3$-only proteins to the mitochondrial surface. This leads to subsequent displacement of further pro-apoptotic activators and effectors from the pro-survival members, promoting BAX/BAK assembly through the outer mitochondrial membrane. These pore-like structures provoke the cytoplasmic leakage of cytochrome $\mathrm{c}$ and other apoptogenic factors, which in turn activate the executioner caspases, and disrupt the mitochondrial transmembrane potential that is necessary for oxidative phosphorylation (OxPHOS). ${ }^{8}$ BH3-only proteins have different interaction modalities for each anti-apoptotic member. BAD, HRK and NOXA bind preferentially to selected anti-apoptotic members, whereas BIM, BID, PUMA and BMF interact indiscrimi- nately with all of them. BAD binds BCL-2, BCL-w and BCL-xL, HRK binds BCL-xL, while NOXA preferentially binds MCL- 1 and BFL- $1 .{ }^{6}$

In hematologic malignancies, there is selective pressure for upregulating the pro-survival members via genetic and non-genetic mechanisms. For example, in both follicular lymphoma (FL) and double-hit diffuse large-B cell lymphoma (DLBCL), $t(14 ; 18)$ juxtaposes $B C L 2$ to the immunoglobulin heavy chain (IGH) locus, increasing BCL-2 protein levels.,10 In chronic lymphocytic leukemia (CLL) with del(13q), the lack of microRNA 15/16 derepresses BCL-2 expression. ${ }^{11}$ Multiple myeloma (MM) and selected subtypes of DLBCL harbor the 1q21 amplification, which leads to MCL-1 overexpression. ${ }^{12,13}$ On the other hand, pro-apoptotic BH3-only members are sometimes downregulated. BIM is epigenetically silenced in a subset of patients with acute lymphoblastic leukemia (ALL), leading to glucocorticoid resistance and poorer outcomes. ${ }^{14}$ Similarly, a subgroup of patients with

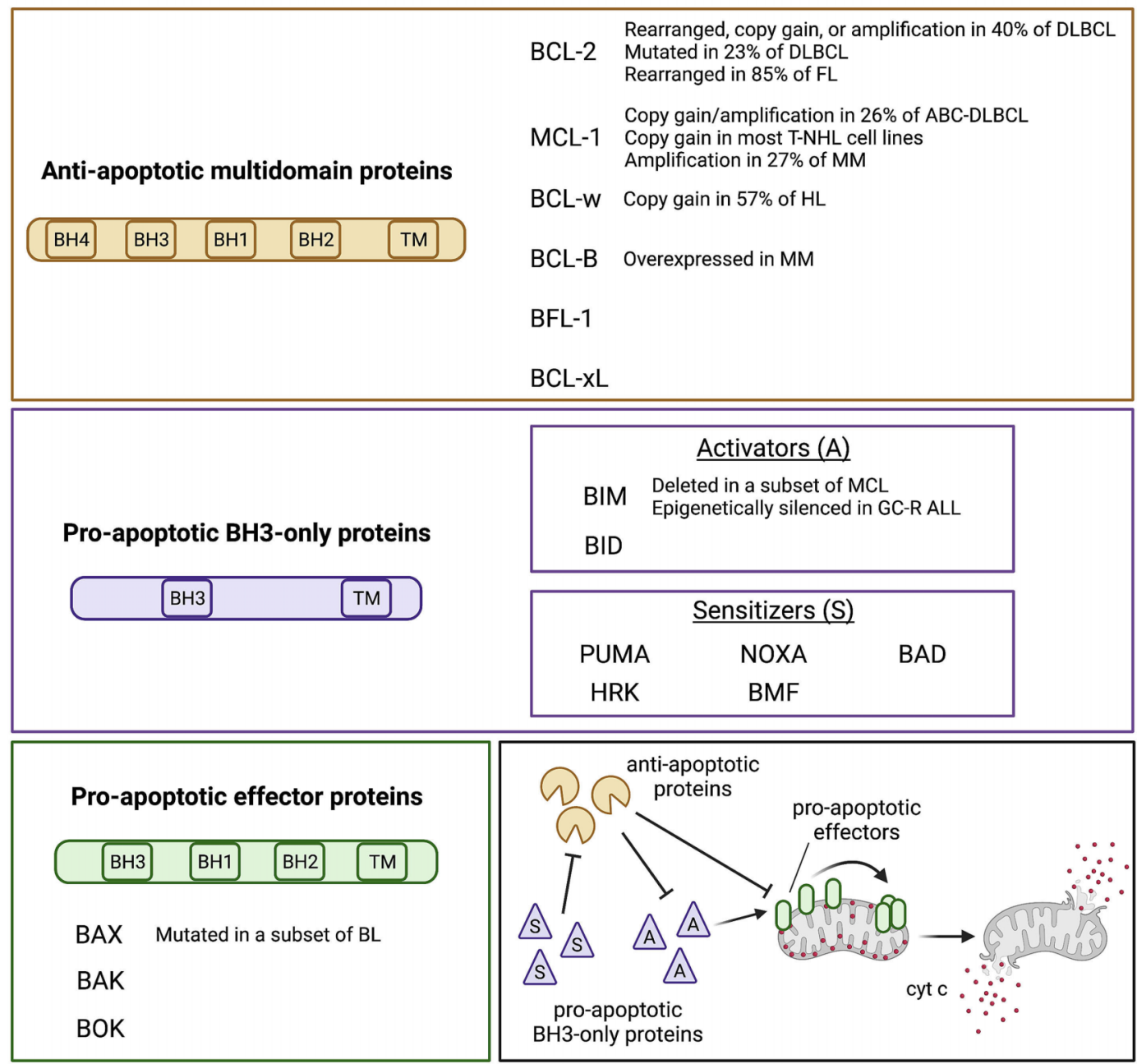

Figure 1. The BCL-2 protein family. The three subgroups of the BCL-2 protein family and their recurrent genetic alterations are shown in the colored boxes. A schematic of the interactions occurring among the BCL-2 family members is represented in the lower right box, based on the "indirect activation" model. Overall, the antiapoptotic proteins sequester the pro-apoptotic BH3-only activators (A) and effectors, preventing the initiation of the apoptotic cascade. By contrast, the pro-apoptotic sensitizers (S) antagonize the anti-apoptotic members thus freeing the activators, which in turn trigger the polymerization of the effectors. This creates pore-like structures at the outer mitochondrial membrane which favor the release of cytochrome c (cyt c) and other apoptogenic factors. While the model shown here has been widely adopted to define the concept of apoptotic priming, recent evidence suggests that, at least in selected cancer types, BAX and BAK activation may only require that these members are freed from the anti-apoptotics, with no need of direct interaction with pro-apoptotic members. DLBCL: diffuse large B-cell lymphoma; FL: follicular lymphoma; ABC: activated B-cell; T-NHL: T-cell non-Hodgkin lymphoma; MM: multiple myeloma; HL: Hodgkin lymphoma; MCL: mantle cell lymphoma; GC-R: ALL glucocorticoid-resistant acute lymphoblastic leukemia; BL: Burkitt lymphoma. 
mantle cell lymphoma expresses low levels of BIM and is less likely to achieve complete response to standard treatments. ${ }^{15}$ All of these mechanisms converge towards apoptosis evasion, a common denominator for cancer cells.

\section{Evasion from mitochondrial apoptosis}

Solid tumors and hematologic malignancies evade mitochondrial apoptosis in markedly different ways (Figure 2), perhaps reminiscent of the biology of the normal tissue counterparts. ${ }^{16}$ Diseases such as kidney, colorectal, and cervical cancer show an extremely poor proclivity to activate intrinsic apoptosis, even when strong pro-apoptotic stressors are directly applied on cancer cell mitochondria. Such low apoptotic priming, defined by the near absence of BH3-only activators on the mitochondrial surface, is a major factor contributing to the chemoresistance of solid tumors, and suggests targeting solely this pathway might not be successful against these diseases. ${ }^{16}$ By contrast, mitochondria of blood cancer cells struggle to maintain the integrity of the outer mitochondrial membrane due to its occupation by several proapoptotic activators (i.e., high mitochondrial priming). In this type of cancers, apoptotic evasion is based on the activity of several anti-apoptotic proteins aiming at buffering large amounts of pro-apoptotics that dynamically shuttle between the cytoplasm and the mitochondria. ${ }^{16}$ The need for an efficient anti-apoptotic arsenal creates a specific vulnerability that is being successfully targeted by venetoclax and other BH3 mimetics. ${ }^{17}$ This class of small molecules targets the interaction interface between the anti- and pro-apoptotic members thus allowing the latter to initiate the apoptotic cascade. While in some cases a single anti-apoptotic protein is the only barrier to apoptotic triggering, in others multiple antiapoptotic members act in concert to oppose outer mitochondrial membrane permeabilization ${ }^{1820}$ (Figure 2).

\section{Genetic and functional approaches to detect anti-apoptotic dependencies}

Over the last 15 years, several genetic and functional methodologies have been set up to derive anti-apoptotic dependencies in cancer. Genetic knock-out of selected anti-apoptotic proteins using CRISPR-Cas9 or related gene-editing techniques have pointed out the anti-apoptotic role of MCL-1 and BCL-w in myc-driven lymphomas. ${ }^{21-23}$ Moreover, doxycycline-inducible silencing RNA (siRNA) targeting specific pro-survival proteins has been successfully transfected in human lymphoma cell lines to evaluate which member has the greatest impact on in vitro cell survival. ${ }^{24}$ While the strength of these approaches lies in their ability to accurately inform about the role of a selected BCL-2 family gene, they are poorly applicable to primary cells from cancer patients. As their use is mostly limited to cancer cell lines, genetic approaches are precious to infer general principles of apoptotic regulation in a given cancer type, but lack scalability to large numbers of patient-derived samples. This is of relevance because some of the most common hematologic malignancies such as acute myeloid leukemia (AML) and DLBCL show heterogeneity of anti-apoptotic dependencies across patients, and perhaps even within patients over the course of their disease..$^{19,25,26}$

Functional approaches provide higher scalability but less molecular insight. They are mainly based on in vitro and ex vivo pharmacological targeting of pro-survival $\mathrm{BH} 3$ proteins, and on BH3 profiling. .7 $^{2-30}$ Side-by-side comparisons of different $\mathrm{BH} 3$ mimetics targeting distinct antiapoptotic proteins have been performed on cancer cell lines and primary samples, and several readouts such as intracellular ATP content, annexin V externalization, and caspase 3/7 activation have been utilized to detect differences in cell viability. 27,28 These pharmacological assays inform about the anti-apoptotic dependencies of tumor samples, working at the same time as apoptosis-tailored drug sensitivity screens with potential impact on preci-

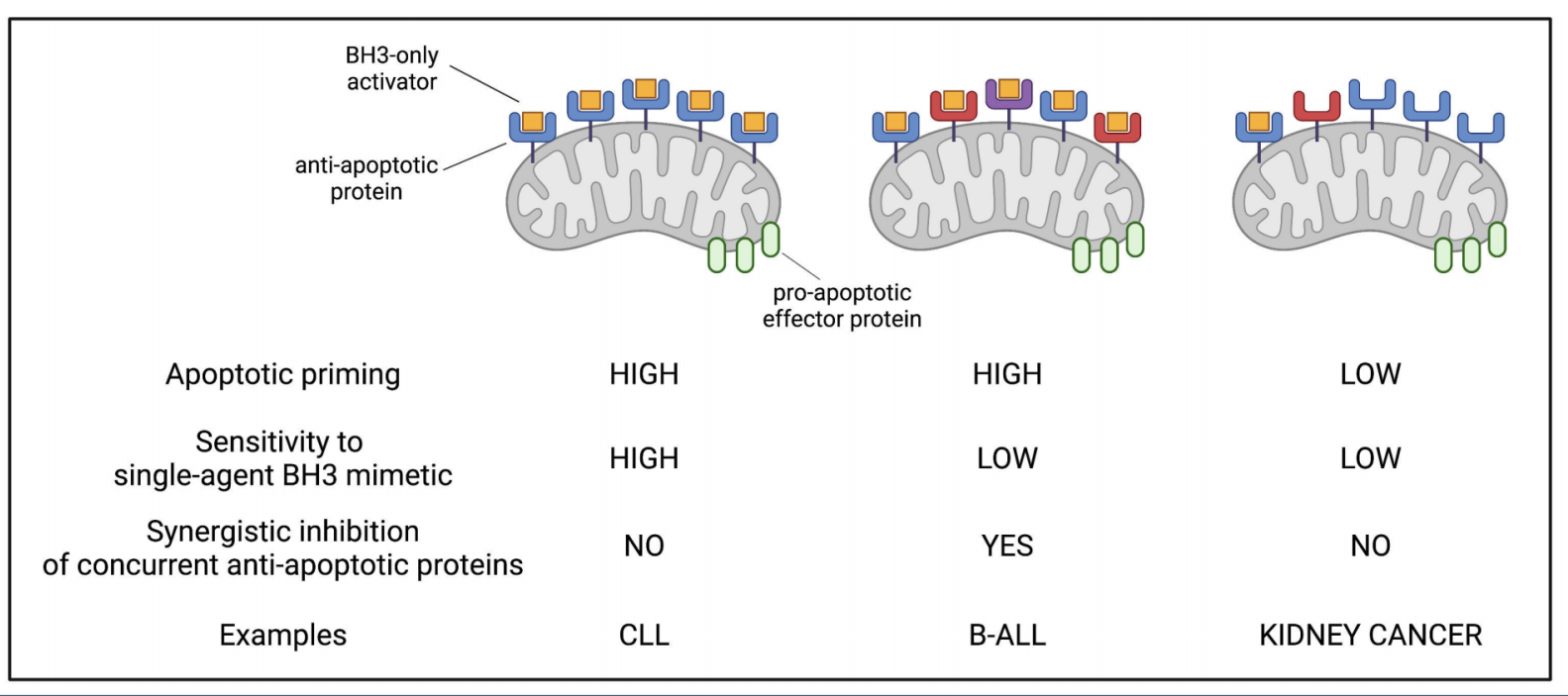

Figure 2. Different forms of evasion from mitochondrial apoptosis. Three distinct scenarios are depicted. In cancers such as chronic lymphocytic leukemia, large amounts of pro-apoptotic activators are sequestered (i.e., high priming) by a single anti-apoptotic relative. In other hematologic cancers (e.g., B-cell acute lymphoblastic leukemia), apoptotic priming is high, but the pro-apoptotics are concurrently sequestered by multiple anti-apoptotic relatives (e.g., BCL-2, MCL-1 and BCL-xL). In solid tumors, pro-apoptotic members are mostly not bound to the anti-apoptotic proteins, and hence sensitivity to BH3 mimetics is generally low. CLL: chronic lymphocytic leukemia; B-ALL: B-cell acute lymphoblastic leukemia. 
sion strategies. Long BH3-mimetic incubation times (i.e., more than 18-24 hours), which may be needed to detect many of the cell-death readouts, is a common shortcoming of these assays as primary cells do not often survive long-term in ex vivo cultures. ${ }^{31}$ Moreover, it has been reported that in vitro-cultured primary cancer cells lose similarities with the original tumor over time, ${ }^{32}$ thus weakening the reliability of the results. Off-target effects of some $\mathrm{BH} 3$ mimetics, for example BCL-2-independent inhibition of OxPHOS by venetoclax, ${ }^{33}$ might be an additional limitation of these assays when the primary aim is to study the biology of the disease rather than the drug efficacy. A clinically applicable declination of such approaches, called the BH3-mimetic toolkit, has been employed on MM samples using CD138 loss as a flow cytometry readout of cancer cell death. Venetoclax (a BCL-2 inhibitor), A1155463, A1331852 (both BCL-xL inhibitors), and A1210477 (a MCL-1 inhibitor) were tested at multiple concentrations, and three dependency groups were derived in an unbiased way using analytical tools. ${ }^{30} \mathrm{BH} 3$ profiling is a different functional technique based on exposing cancer cell mitochondria to an array of pro-apoptotic peptides with distinct binding modalities for different anti-apoptotic members. In this assay, the anti-apoptotic addiction of cancer cells can be inferred by the pattern of cytochrome $c$ release upon peptide incubation $^{34}$ Due to the short incubation time, BH3 profiling averts the risk of artifactual genetic selections or functional modifications that could potentially occur during prolonged ex vivo culture. In addition, the specificity of treating peptides that act directly on the mitochondrial surface renders this assay particularly focused on the BCL-2 fam- ily dynamics, without off-target effects that might instead be encountered using small molecules. A potential downside is that BH3 profiling is an organelle-centered method that does not take into account how other cellular components might react to peptide-induced cytochrome c release. For instance, defective activation of downstream cytosolic caspases, which sometimes occurs in solid tumors due to somatic mutations, ${ }^{35}$ may blunt the apoptotic response triggered by cytochrome c leakage. $\mathrm{BH} 3$ profiling also functions as a platform to set up an additional assay, named dynamic BH3 profiling, which measures changes in apoptotic priming and anti-apoptotic dependencies triggered by drug candidates. In this case, the incubation with pro-apoptotic peptides is preceded by ex vivo treatment with a panel of drugs, with the aim of identifying those that most efficiently lower the threshold for cytochrome c release..$^{36,37}$ Dynamic BH3 profiling has proven useful to assess the impact of Bruton tyrosine kinase (BTK) inhibitors on BCL-2 dependence of CLL cells, ${ }^{38}$ and as a functional precision medicine strategy for T-cell prolymphocytic leukemia. ${ }^{39}$

\section{The anti-apoptotic map of hematologic malignancies}

Extensive preclinical research and clinical observations are building our knowledge on how cancer cells evade apoptosis. The anti-apoptotic map of hematologic malignancies (Figure 3) has recently guided highly effective treatments for CLL and AML, ${ }^{40,41}$ and is currently inspiring novel antineoplastic regimens for other types of blood

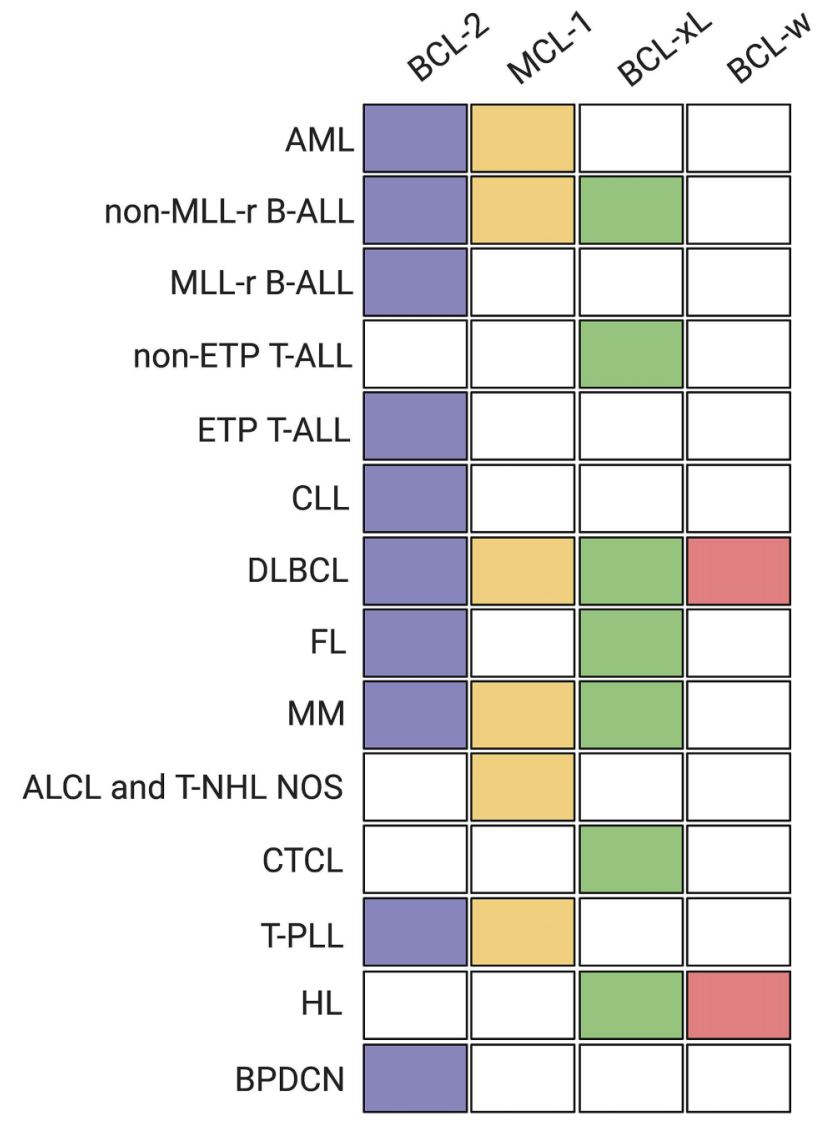

Figure 3. The anti-apoptotic map of hematologic malignancies. The colored map shows the antiapoptotic dependencies of several hematologic $\begin{array}{ll}41,43,47 & \begin{array}{l}\text { malignancies, based on preclinical data and clini- } \\ \text { cal results (see references). With the exception of } \\ \text { follicular lymphoma, in which the BCL-xL depen- } \\ \text { dence is driven by microenvironmental stimuli, all }\end{array}\end{array}$ $\begin{array}{ll}41,43,47 & \begin{array}{l}\text { malignancies, based on preclinical data and clini- } \\ \text { cal results (see references). With the exception of } \\ \text { follicular lymphoma, in which the BCL-xL depen- } \\ \text { dence is driven by microenvironmental stimuli, all }\end{array}\end{array}$ $\begin{array}{ll}41,43,47 & \begin{array}{l}\text { malignancies, based on preclinical data and clini- } \\ \text { cal results (see references). With the exception of } \\ \text { follicular lymphoma, in which the BCL-xL depen- } \\ \text { dence is driven by microenvironmental stimuli, all }\end{array}\end{array}$ the other anti-apoptotic dependencies depicted here refer to cell-intrinsic dependencies. AML: acute myeloid leukemia; MLL-r B-ALL: MLL-rearranged $\mathrm{B}$-cell acute lymphoblastic leukemia; ETP T-ALL: early T-cell acute lymphoblastic leukemia; CLL: chronic lymphocytic leukemia; DLBCL: diffuse large B-cell lymphoma; FL: follicular lymphoma; MM multiple myeloma; ALCL: anaplastic large T-cell lymphoma; T-NHL NOS: T-cell nonHodgkin lymphoma not otherwise specified; CTCL: cutaneous T-cell lymphoma; T-PLL: T-cell prolymphocytic leukemia; HL: Hodgkin lymphoma; BPDCN: blastic plasmacytoid dendritic cell neoplasia.

$19,26,65$

\section{1}

$12,20,30$

80,81

80

39,83 
disorders. This section focuses on key preclinical findings relevant to apoptosis avoidance. Clinical results of $\mathrm{BH} 3$ mimetics in hematology will only be touched on, as they have been recently reviewed by Roberts and colleagues. ${ }^{42}$

\section{Acute myeloid leukemia and myelodysplastic syndromes}

Regulation of intrinsic apoptosis in AML shows both intratumor and interpatient heterogeneity. In 2014, Pan and co-workers identified a BCL-2 dependence for roughly $80 \%$ of primary AML cases, with rapid apoptotic triggering upon ex vivo exposure to venetoclax. BCL-2 protein expression correlated with sensitivity to venetoclax, whereas BCL-xL and, to a lesser extent MCL-1, showed anti-correlation with susceptibility to BCL-2 inhibition. ${ }^{43}$ A phase II trial evaluating the activity of venetoclax as a single agent in high-risk AML demonstrated an overall response rate of $19 \%$, with particularly favorable responses among patients carrying isocitrate dehydrogenase 1/2 (IDH1/2) mutations. ${ }^{41}$ BH3 profiling identified patients who were more likely to stay on venetoclax therapy longer than 30 days, working as a predictive functional assay with potential clinical applications. ${ }^{41}$ Bone marrow cells from patients with high-risk myelodysplastic syndromes are also sensitive to BCL-2 antagonism. In vitro treatment with $\mathrm{ABT}-737$ or venetoclax depletes the myelodysplastic syndrome progenitor compartment, and decreases the colony-forming capacity and the percentage of $\mathrm{CD} 34^{+}$cells. ${ }^{44}$ The combination of venetoclax plus intensive chemotherapy led to complete remission in $82 \%$ of patients with newly-diagnosed AML and highrisk myelodysplastic syndromes ${ }^{45}$ Despite the meaningful clinical activity of venetoclax-based regimens in these settings, about $20 \%$ of patients are primarily refractory. Among AML patients achieving complete remission with venetoclax plus azacytidine, the median duration of response was only 11.3 months. ${ }^{46}$ This suggests that a subset of AML cases is not BCL-2 dependent, and that additional groups of patients may harbor subclonal dependencies to different anti-apoptotic proteins. A recent study addressed this point and found that BCL-2 dependence decreases through stages of AML morphological maturation. ${ }^{47}$ Indeed, lower BCL-2 expression, at both mRNA and protein levels, was observed in FrenchAmerican-British (FAB) M5 AML compared to FABM0/M1/M2 leukemia. A combination of venetoclax plus azacitidine failed to inhibit OxPHOS in monocytic AML, with a modest impact on cell viability. ${ }^{47}$ By contrast, the MCL-1 inhibitor VU661013 combined with azacitidine significantly suppressed OxPHOS in monocytic cases and was more effective than venetoclax-based treatments in inducing cell death. Genetic knockdown of MCL-1 was sufficient to trigger apoptosis in primary monocytic AML specimens, further indicating their reliance on MCL-1 to maintain survival. ${ }^{47}$ Accordingly, $62 \%$ of patients with FAB-M5 AML were refractory to venetoclax-azacitidine, whereas only $8 \%$ of non-FAB-M5 cases did not respond to this regimen. ${ }^{47} \mathrm{~A}$ side-by-side comparison of $\mathrm{BH} 3$ mimetics in mediating AML cell killing confirmed that a subset of immortalized and primary AML cells is highly sensitive (low micromolar/nanomolar range) to the MCL1 inhibitor S63845, but not to BCL-xL or BCL-2 inhibitors. ${ }^{27}$

\section{Acute lymphoblastic leukemia}

B-cell ALL shows concurrent dependence on BCL-2 and
BCL-xL, with MCL-1 being identified as a further antiapoptotic member able to confer resistance to single or dual BCL-2/BCL-xL antagonism. ${ }^{48-50}$ Indeed, sensitivity to venetoclax, which was predicted by $B C L 2$ gene expression level as well as BH3 profiling, was highly heterogeneous in a panel of B-ALL cell lines and patient-derived xenograft, with $\mathrm{EC}_{50}$ values ranging from $1.8 \mathrm{nM}$ to 5.5 $\mu \mathrm{M} .{ }^{49}$ Accordingly, venetoclax was effective in vivo in only a minority of B-ALL xenografts, whereas combined inhibition of BCL-2 and BCL-xL resulted in synergistic killing of most B-ALL in vivo models..$^{51} \mathrm{~A}$ recently reported phase I trial of the combination of venetoclax with low-dose navitoclax (dual BCL-2/BCL-xL inhibitor) plus chemotherapy in relapsed/refractory (R/R) B-ALL has shown encouraging results, with a complete remission rate of $60 \% .^{52}$ Despite the heterogeneity and the high degree of anti-apoptotic co-dependencies in B-ALL, the subgroup carrying the $\mathrm{t}(4 ; 11)$ translocation proved uniformly BCL-2-dependent ${ }^{51,53}$ The fusion protein MLL/AF4 activates $B C L 2$ transcription via DOT1L-mediated H3K79me2/3, without altering the expression level of other anti-apoptotic members. This renders MLL-rearranged cells susceptible to venetoclax-induced apoptosis in vitro, and sensitive to venetoclax-based combinations in vivo. ${ }^{53}$

The anti-apoptotic dependency of T-ALL reflects the maturation stage of lymphoblasts. Early T-cell progenitor (ETP) ALL cells most closely resemble early thymic, CD4 /CD8- T cells, and are dependent upon BCL-2. ${ }^{54}$ Instead, non-ETP T-ALL cells have a gene expression and phenotypic profile resembling that of more mature, $\mathrm{CD}^{+} / \mathrm{CD} 8$ $\mathrm{T}$ cells, expressing abundant BCL-xL protein levels, and having functional dependency upon BCL-xL. ${ }^{54}$ While ETP-ALL patient-derived xenograft models are very sensitive to venetoclax in vivo, the non-ETP counterpart is relatively resistant. ${ }^{54}$ Moreover, sensitivity to long-term BCL-2 inhibition in ETP-ALL might be compromised by microenvironment-derived signals. More specifically, the spleen has been identified as a sanctuary site for residual ETP lymphoblasts following venetoclax treatment. Such surviving cells display decreased BCL-2 expression and reduced BCL-2 dependence, with requirement of concomitant MCL-1 inhibition to evoke robust cell death. ${ }^{55}$

\section{Chronic lymphocytic leukemia}

In the light of its broad heterogeneity in terms of chromosomal aberrations, gene mutations, clinical characteristics, and drug response profiles, ${ }^{56-58}$ CLL shows surprisingly homogeneous anti-apoptotic regulation..$^{18}$ More than three decades of basic discoveries have pointed out that BCL-2 is overexpressed in CLL cells compared to normal B lymphocytes due to gene promoter hypomethylation, ${ }^{59} \mathrm{miR} 15 / 16$ downregulation, ${ }^{11}$ and, more rarely, BCL2 translocation. ${ }^{60}$ Such genetic bases for BCL-2 addiction have been functionally confirmed by $\mathrm{BH} 3$ profiling, which revealed a clear-cut BCL-2 dependence of CLL cells regardless of TP53 status and previous lines of therapy. ${ }^{18,61}$ This set the stage for the clinical introduction of venetoclax, the first approved selective BCL-2 inhibitor, which achieved an overall response rate of $79 \%$ with $20 \%$ of complete remissions in the R/R CLL setting..$^{40}$

While cell-intrinsic genetic programs seem to be responsible for the exceedingly high BCL-2 dependency of CLL cells, signals from the microenvironment can add further layers of anti-apoptotic protection. Indeed, anti- 
gen stimulation and CXCL12, both converging on BTK, decrease apoptotic priming and BCL-2 dependence. ${ }^{62}$ As a consequence, ibrutinib and other inhibitors of the B-cell receptor signaling pathway increase BCL-2 dependence by impeding extrinsic signals to upmodulate MCL-1 and BCL-xL which would ultimately favor additional pro-survival forces. ${ }^{38}$ The CLARITY study, exploring the combination of ibrutinib and venetoclax in R/R CLL, found $51 \%$ complete remissions with a high rate of minimal residual disease eradication, which enabled treatment cessation in a subset of patients. ${ }^{63}$

\section{B-cell non-Hodgkin lymphoma}

DLBCL, the most common type of B-cell nonHodgkin lymphoma (NHL), is remarkably heterogeneous in terms of mutational landscape and clinical pictures. ${ }^{64}$ Anti-apoptotic dependencies show heterogeneity as well, and do not correlate with cell of origin. ${ }^{19,26,65}$ Loss of the anti-apoptotic BCL-w was reported to delay MYC-driven lymphoma development in E $\mu$-Myc transgenic mice by augmenting MYC-induced apoptosis. ${ }^{22}$ Moreover, BCL-w is overexpressed in a subset of DLBCL characterized by shorter overall survival, suggesting a primary role for apoptosis evasion. ${ }^{22}$ Despite this, a recent study found that CRISPR/Cas9-mediated loss of BCL-w did not trigger apoptosis in DLBCL cell lines, nor did it increase the sensitivity to $\mathrm{BH} 3$ mimetics targeting additional pro-survival proteins, casting doubts about the role of BCL-w in human DLBCL. ${ }^{28}$ MYC-driven lymphomagenesis is also sustained by MCL-1, which is highly expressed in activated B-cell DLBCL. ${ }^{66}$ MCL1 copy number abnormalities were detectable in $25.7 \%$ of activated B-cell -DLBCL versus $12.5 \%$ of germinal-center DLBCL, and constitutive STAT3 signaling further contributes to MCL-1 upregulation in selected cases. ${ }^{6}$ The MCL-1 antagonist AZD5991, currently in clinical testing, curtails tumor growth and disrupts mitochondrial metabolism in MCL-1-addicted DLBCL cells via TP53- and BAXdependent mechanisms. ${ }^{13}$ BCL2 is also frequently deregulated in DLBCL due to the $\mathrm{t}(14 ; 18)$ chromosomal translocation, gene mutations, copy number alterations and amplifications. ${ }^{67}$ Such genetic events confer poor prognosis especially when combined with MYC amplification. ${ }^{10}$ While in some cases BCL2 dysregulation is associated with functional dependency on BCL-2 and high sensitivity to venetoclax, in others targeting additional pro-survival members is needed to achieve apoptosis. Indeed, several B-cell receptor-dependent DLBCL lines with genetic bases for BCL-2 dysregulation require dual targeting of $\mathrm{PI} 3 \mathrm{~K} \alpha / \delta$, which in turns decreases MCL-1 abundance, and BCL-2 to commit cell death. ${ }^{68}$ Anti-apoptotic heterogeneity and frequent co-dependencies account for the disappointing results of singleagent venetoclax against DLBCL, with an overall response rate of only $18 \%$ in $\mathrm{R} / \mathrm{R}$ cases. ${ }^{69}$ Less common subtypes of B-NHL, including follicular lymphoma and mantle cell lymphoma, display better clinical responses to BCL-2 inhibition. ${ }^{69,70}$ However, durable remissions are not frequent and may require combination therapies. At least in follicular lymphoma, in which high BCL-2 expression caused by the $t(14 ; 18)$ translocation has ever been considered the pathogenic hallmark, concurrent antagonism of microenvironment-induced BCL$\mathrm{xL}$ is needed to efficiently trigger apoptosis. ${ }^{71}$

\section{Multiple myeloma}

At least one-third of $\mathrm{MM}$ cases are predominantly MCL-1 dependent, whereas the others are either BCL-2 dependent or characterized by BCL-2/MCL-1 co-dependence. . $2,20,30,72$ Moreover, a minority of MM cell lines and primary samples show some degree of BCL-xL dependence. ${ }^{30,72} \mathrm{MCL}-1$ addiction can be driven by genetic alteration and microenvironmental cues. The MCL1 gene is located on 1q21, which is amplified in $43-72 \%$ of MM patients depending on disease status. ${ }^{73}$ 1q21 copy gain directly correlates with MCL1 transcript abundance and MCL-1 protein expression. ${ }^{12}$ Importantly, plasma cells from 1q21-amplified cases are especially sensitive to MCL-1 inhibition, while proving relatively resistant to venetoclax. ${ }^{12}$ Interleukin- 6 released by bone marrow stromal cells can further amplify MCL-1 dependence through MCL-1 transcriptional upregulation and BIM phosphorylation. Such events switch the BIM binding partner from BCL-2 and BCL-xL to MCL-1. ${ }^{747}$ Blocking the interleukin6 signaling pathway decreases MCL-1 dependence and enhances sensitivity to venetoclax. ${ }^{76,77}$ On the other hand, BCL-2-dependent cases are enriched among MM with the $\mathrm{t}(11 ; 14)$ (q13; q32) translocation, which is detected in $15 \%$ to $20 \%$ of cases. $^{78}$ In the phase I study of venetoclax monotherapy in $\mathrm{R} / \mathrm{R} \mathrm{MM}$, the overall response rate was $21 \%$ in the whole population, but reached up to $40 \%$ in the subgroup harboring $t(11 ; 14) .{ }^{79}$ High BCL2:MCL1 and BCL2:BCL2L1 mRNA expression ratios correlated with venetoclax sensitivity. ${ }^{79}$ Given the anti-apoptotic heterogeneity and the frequent co-dependencies of MM cells, dual targeting of BCL-2 and MCL-1 has been assessed in preclinical studies, showing a profound synergism potentially translatable to the clinic. ${ }^{77}$ Moreover, the $\mathrm{BH} 3-$ mimetic toolkit revealed an increase in MCL-1 addiction from $33 \%$ at diagnosis to $69 \%$ at relapse, indicating temporal remodeling of cellular dependencies. ${ }^{30}$ This assay also identified a subset of newly diagnosed $\mathrm{MM}$ patients not sensitive to any of the $\mathrm{BH} 3$ mimetics, highlighting that apoptosis targeting might not always be a suitable therapeutic option. ${ }^{30}$

\section{T-cell non-Hodgkin lymphoma}

Protein expression analyses identified MCL-1 as the major anti-apoptotic member in cell lines and primary samples of systemic T-cell NHL. ${ }^{80,81}$ Copy number gains involving the MCL1 locus were found in ten out of $21 \mathrm{~T}$ NHL cell lines. ${ }^{80}$ Accordingly, loss of a single MCL1 allele delayed tumor formation in T-NHL mouse models and compromised the viability of neoplastic $\mathrm{T}$ cells, ${ }^{81} \mathrm{BH}$ profiling confirmed that most T-NHL cell lines, especially those from anaplastic T-cell lymphomas and other peripheral T-cell lymphomas, are MCL-1 dependent. ${ }^{80}$ The development of several MCL-1 antagonists has recently allowed the translation of these biological findings into pharmacological approaches. Indeed, AZD5991 reduced tumor volumes in vivo, and synergized with cyclophosphamide, vincristine, doxorubicin and prednisone to improve survival of mice with T-NHL..$^{80}$ By contrast, cutaneous T-NHL cases are primarily BCL-xL dependent, and some of them carry copy number alteration of the BCL2L1 gene. $^{80} \mathrm{~A}$ proteolysis targeting chimera that targets BCL-xL for degradation has been developed to effectively kill cutaneous T-NHL cells in vitro and in vivo, without causing significant thrombocytopenia as previously reported for navitoclax. ${ }^{82}$ 


\section{T-cell prolymphocytic leukemia}

As compared to CLL, T-cell prolymphocytic leukemia is less primed for apoptosis, less BCL-2 dependent, and often BCL-2/MCL-1 co-dependent. ${ }^{39,83}$ BCL-xL dependence is usually more pronounced in T-cell prolymphocytic leukemia than in CLL, but remains of secondary importance when compared with BCL-2 and MCL-1 dependence. ${ }^{39}$ Clinical reports suggest that venetoclax monotherapy is often inadequate to get durable disease control. ${ }^{84}$ As demonstrated by dynamic BH3 profiling, inhibitors of histone deacetylase and the JAK/STAT pathway increase apoptotic priming and BCL-2 dependence, strengthening the pro-apoptotic effect of venetoclax in vitro and in vivo. ${ }^{39}$

\section{Hodgkin lymphoma}

As compared to normal B lymphocytes, Hodgkin lymphoma cells express higher levels of BCL-w and BCL-xL mRNA. ${ }^{85}$ BCL-w expression further increases in advanced stages and in the R/R setting. ${ }^{85}$ Fluorescence in situ hybridization analyses on Hodgkin lymphoma samples showed that copy number gains of chromosome 14 and amplifications of the BCL-w containing region were common events in the pathogenesis of Hodgkin lymphoma and led to almost invariably high BCL-w protein expression as detected by immunohistochemistry. Genetic knockdown of BCL-w or pharmacological antagonism of BCL-xL significantly reduced Hodgkin lymphoma cell viability, pointing to a primary role for these anti-apoptotic proteins in sustaining Hodgkin lymphoma growth. ${ }^{85}$

\section{Blastic plasmacytoid dendritic cell neoplasm}

Preclinical data indicate that blastic plasmacytoid dendritic cell neoplasm is primarily BCL-2 dependent. Blastic plasmacytoid dendritic cell neoplasm cells collected from patient-derived xenografts or directly from patients' skin and bone marrow showed higher BCL-2 dependency compared with randomly selected AML cases. ${ }^{86}$ In vivo experiments confirmed these findings and, to date, several reports have been published about patients with relapsed blastic plasmacytoid dendritic cell neoplasm successfully treated with single-agent venetoclax. ${ }^{87,88}$ Such a therapeutic approach is currently being investigated in a phase I clinical trial (NCT03485547).

\section{Resistance to BH3 mimetics: a tale of cellular interactions, mitochondrial biology and mutational pressure}

With the growing use of venetoclax in clinical practice, several resistance mechanisms have been outlined, especially in the context of CLL and AML which currently represent the major indications for BCL-2 inhibition. Three broad concepts are emerging. First, clinical progressions on venetoclax are mostly underpinned by "polyclonal patterns", whereby different leukemic clones within the same

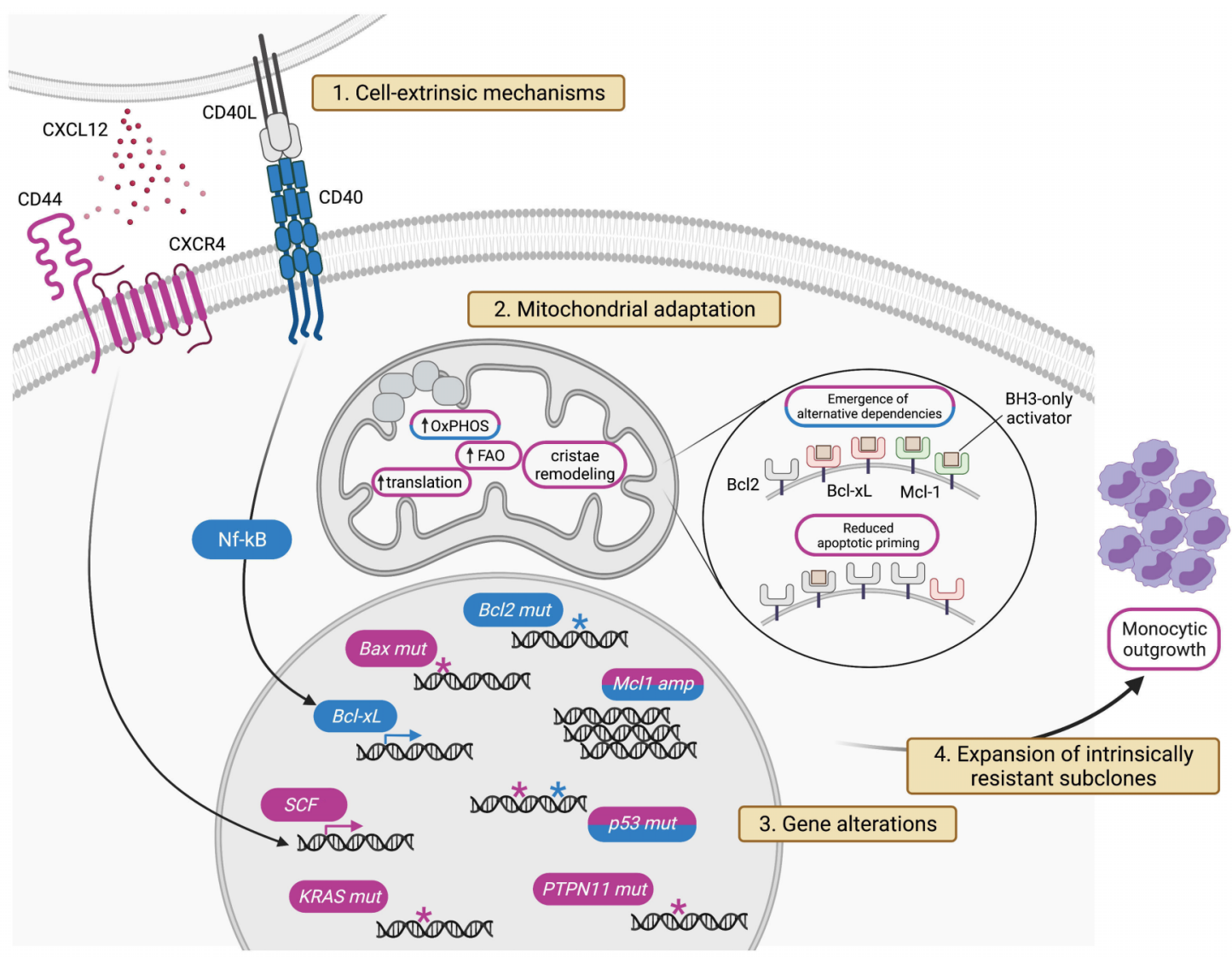

Figure 4. Mechanisms of venetoclax resistance. The figure depicts the four major modalities of resistance to venetoclax: cell-extrinsic mechanisms, outer and inner mitochondrial adaptation, genomic alterations, and expansion of intrinsically resistant subclones. Mechanisms highlighted in purple have been described in acute myeloid leukemia. Those highlighted in blue have been reported in chronic lymphocytic leukemia. See text for pathway details. SCF: stem cell factors; OxPHOS: oxidative phosphorylation; FAO: fatty acid oxidation. 
Table 1. Treatment strategies that may prevent/overcome escape from venetoclax based on preclinical mechanistic predictions.

\begin{tabular}{|c|c|c|c|c|}
\hline Treatment strategy & Indication & Mode of action of partner drug & Study phase & NCT number \\
\hline \multicolumn{5}{|c|}{ Escape mechanism: extrinsic interactions and downstream signaling pathways } \\
\hline cirmtuzumab + venetoclax & CLL & anti-ROR1 & I & NCT03797261 \\
\hline duvelisib + venetoclax & CLL & PI3K $\gamma \delta$ inhibitor & I/II & NCT03534323 \\
\hline copanlisib + venetoclax & DLBCL & PI3K $\alpha \delta$ inhibitor & I/II & NCT04572763 \\
\hline ruxolitinib + venetoclax & AML & JAK inhibitor & I & NCT03874052 \\
\hline BP1001 + venetoclax & AML & L-Grb2 antisense oligonucleotide & II & NCT02781883 \\
\hline ibrutinib + venetoclax & CLL & covalent BTK inhibitor & II & NCT02756897 \\
\hline loxo305 + venetoclax + rituximab & CLL & non-covalent BTK inhibitor & III & NCT04965493 \\
\hline plerixafor + venetoclax & AML & anti-CXCR4 & / & / \\
\hline
\end{tabular}

\section{Escape mechanism: anti-apoptotic and metabolic mitochondrial adaptation}

\begin{tabular}{lcccc} 
AMG $176+$ venetoclax & R/R heme malignancies & MCL-1 inhibitor & I & NCT03797261 \\
S64315 + venetoclax & AML & MCL-1 inhibitor & I & NCT03672695 \\
\hline AZD5991 + venetoclax & AML & MCL-1 inhibitor & I/II & NCT03218683 \\
Navitoclax + venetoclax + chemo & B-ALL & BCL-xL inhibitor & I & NCT03181126 \\
\hline adi-peg $20+$ venetoclax + azacytidine & AML & arginine depleting enzyme & I & NCT05001828 \\
Omacetaxine + venetoclax & AML & protein translation inhibitor & I & NCT04874194 \\
\hline IACS-010759 + venetoclax & AML & OxPHOS inhibitor & $/$ & $/$ \\
ONC201 + venetoclax & AML & OxPHOS inhibitor & $/$ & $/$
\end{tabular}

\section{Escape mechanism: gene alterations}

BGB-11417

B-cell malignancies BCL-2 inhibitor (active against G101V)

NCT04883957

eprenetapopt + venetoclax

MCL

p53 reactivator

$\begin{array}{ll}\text { I } & \text { NCT04883957 } \\ \text { II } & \text { NCT04990778 }\end{array}$

NCT: National Clinical Trials; CLL: chronic lymphocytic leukemia; DLBCL: diffuse large B-cell lymphoma; AML: acute myeloid leukemia; R/R: relapsed or refractory; ALL: acute lymphoblastic leukemia; MCL: mantle cell lymphoma.

patient take distinct paths to survive BCL-2 inhibition independent of each other. ${ }^{99}$ Secondly, the general biological principles driving resistance to one specific $\mathrm{BH} 3$ mimetic are shared with other BCL-2 family antagonists. Although this section is mainly focused on the mechanisms of resistance to venetoclax, the only Food and Drug Administration-approved $\mathrm{BH} 3$ mimetic so far, early data are piling up about similar escape trajectories occurring in cells treated with MCL-1 or BCL-xL antagonists. ${ }^{25}$ Thirdly, resistance to BH3 mimetics is highly "multimodal", as multiple cellular components can be rewired to undermine the efficacy of BCL-2 family antagonism. The four major modalities of acquisition of resistance to venetoclax are based on cell-extrinsic interactions, ${ }^{90,91}$ mitochondrial adaptation, ${ }^{92}$ genomic alterations, ${ }^{89}$ and, as previously highlighted for the monocytic escape of AML, the emergence of intrinsically resistant clones ${ }^{47}$ (Figure 4). While such variety of resistance mechanisms contrasts with the simpler mutational evolution often observed in patients treated with kinase inhibitors, ${ }^{93}$ it offers several clues to plan at best subsequent therapies, and to design strategic drug combinations as well (Table 1).

\section{Cell-extrinsic interactions}

In AML, CXCL12 released by surrounding stromal cells protects the leukemic clone from BCL-2 inhibition by activating the CD44/CXCR4 complex, which in turn induces the transcription of several pro-survival embryonic stem-cell core transcription factors..$^{90}$ Likewise, in CLL CD40 ligation enhances non-BCL-2 anti-apoptotic dependencies by inducing BCL-xL transcription via the canonical as well as the non-canonical NF-KB pathway. ${ }^{91}$ Induction of pro-survival members by cell-extrinsic factors may account for the relatively less durable response to venetoclax observed among CLL patients with $>5 \mathrm{~cm}$ lymph nodes, where most of the cell-to-cell and paracrine interactions take place. ${ }^{40}$

\section{Mitochondrial adaptations}

Mitochondria are active players in the initiation of venetoclax resistance due to anti-apoptotic remodeling, occurring at the outer mitochondrial membrane, and metabolic reprogramming, occurring at the inner mitochondrial membrane. Functional shifts towards alternative antiapoptotic defenses render leukemic mitochondria progressively less vulnerable to BCL-2 antagonism, with dual BCL-2 and MCL-1 antagonism outperforming the individual targeting. 25,92,94 Moreover, a global reduction of mitochondrial apoptotic priming is quite common in AML acquiring resistance to different types of $\mathrm{BH} 3$ mimetics. ${ }^{25}$ As apoptotic regulation is tightly connected with bioenergetic processes, the upmodulation of mitochondrial metabolic pathways confers resistance to $\mathrm{BH} 3$ mimetics. While in venetoclax-sensitive AML stem cells OxPHOS is suppressed through the inhibition of amino acid metabolism, in resistant clones it is restored through the enhancement of fatty acid oxidation. ${ }^{95}$ In this context, the role of BCL-2 remains unclear because venetoclax is reported to inhibit OxPHOS independently of BCL-2 expression..$^{96}$ Thus, resistant cells might have evolved the ability to restore OxPHOS, bypassing the inhibitory effect of venetoclax. In addition, resistant AML stem cells show elevated nicotinamide metabolism which promotes the uptake and catabolism of amino acids, as well as the conversion of fatty acids into the tricarboxylic acid cycle intermediates 2-oxoglutarate and malate. Indeed, inhibitors of nicotinamide phosphoribosyltransferase specifically target leukemic cells with acquired resistance to venetoclax. ${ }^{97}$ The modulation of mitochondrial cristae ultrastructure also impacts on bioenergetics and $\mathrm{BH} 3$ mimetic sensitivi- 
ty. The mitochondrial chaperonin CLPB has been recently identified as a crucial interactor of the cristae-shaping protein OPA1, and its downregulation alters cristae architecture and renders cytochrome $\mathrm{c}$ more prone for cytosolic release. ${ }^{98}$ An independent genome-wide CRISPR screen identified genes involved in mitochondrial translation as an additional circuit to bypass BCL-2 antagonism, and antibiotics targeting the mitochondrial ribosomes effectively overcome venetoclax resistance. ${ }^{99}$

\section{Genomic alterations}

Emergence of subclones harboring BCL2 mutations is increasingly described in CLL patients progressing on venetoclax, with the most frequent being Gly101Val and substitutions at Asp103. ${ }^{89}$ These BCL-2 structural variants decrease venetoclax binding affinity by several folds. Interestingly, BCL-2 mutants maintain the ability to bind and sequester BIM, thus preserving their fundamental role in the regulation of apoptotic balance. ${ }^{89}$ In other

A

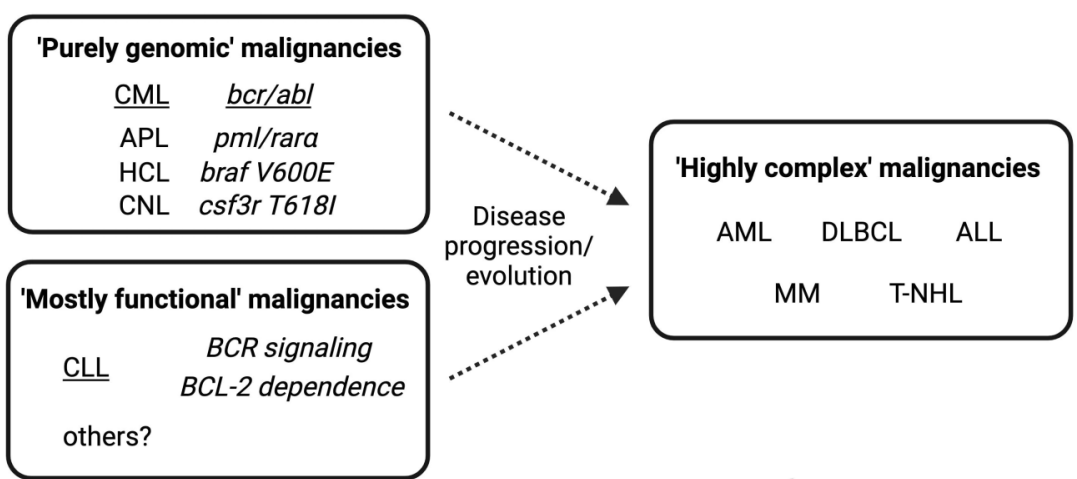

Need for integrated precision medicine

B

The integrated precision medicine approach

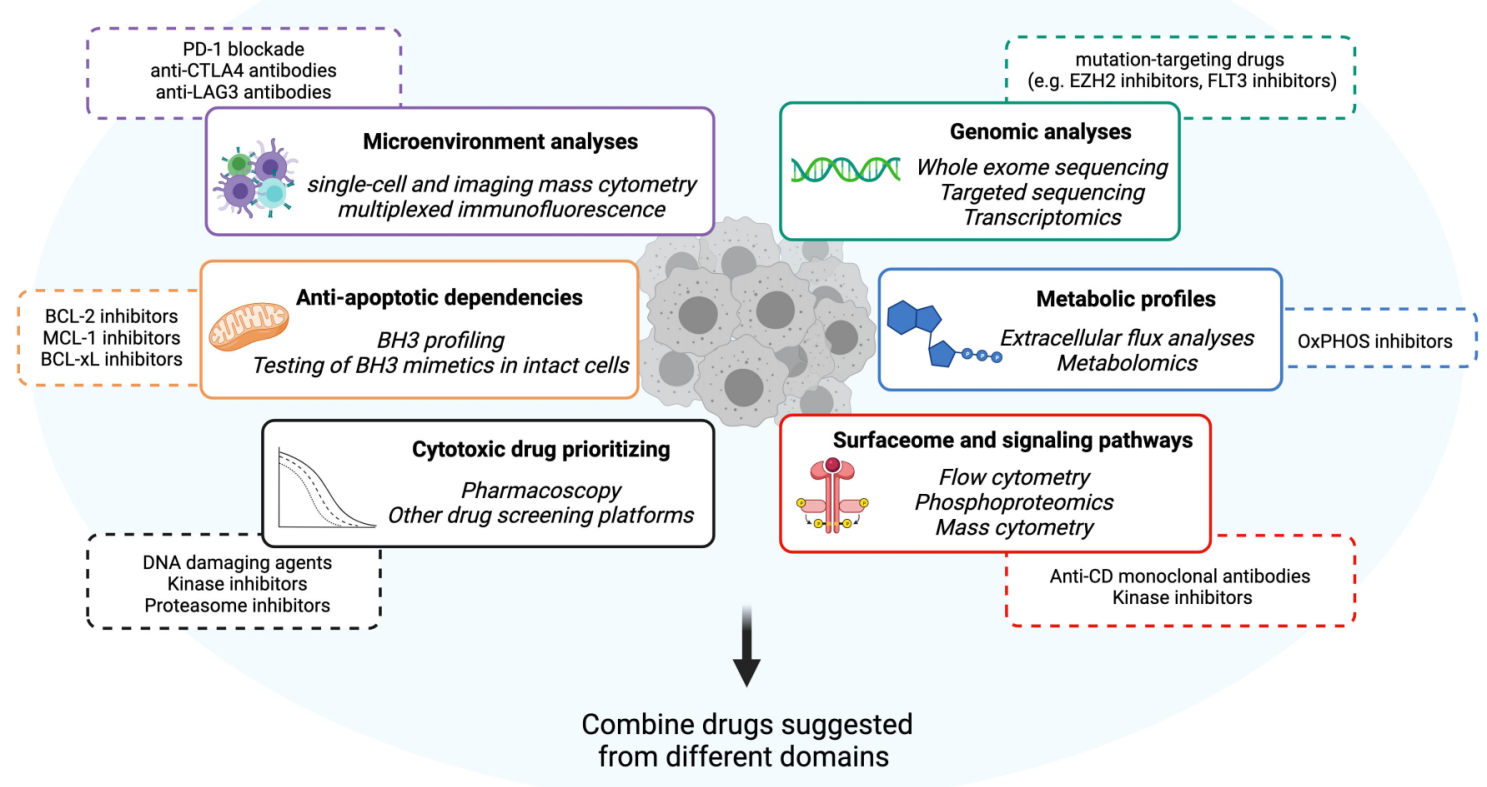

Figure 5. Integrated precision medicine for 'highly complex' hematologic malignancies. (A) Classification of hematologic malignancies based on their molecular/functional complexity and treatment requirements. In chronic myeloid leukemia, the prototype of 'purely genomic' malignancies, a single genomic aberration drives disease biology and treatment modalities. In chronic lymphocytic leukemia, a model for 'mostly functional' malignancies, a few functional pathways (Bcell receptor signaling, BCL-2 dependence) sustain leukemic growth and have proven to be the best drug targets so far. In acute myeloid leukemia and other 'highly complex' malignancies, multiple driver mutations and functional oncogenic pathways co-occur to promote cancer growth and escape treatments. This category might be approached with an integrated precision medicine strategy. (B) Integrated precision medicine is based on interrogation of different static (i.e., microenvironment, surfaceome, genomics) and functional (i.e., anti-apoptotic and metabolic dependencies, signaling pathways, drug sensitivity) domains through dedicated assays (italics). Each of these assays will provide information about different tumor-specific vulnerabilities (e.g., BH3 profiling might highlight BCL-2 dependence, extracellular flux analysis might indicate oxidative phosphorylation utilization, microenvironment analysis might demonstrate CTLA-4-based interactions). Eventually, a combination of drugs targeting vulnerabilities from different domains is suggested, with potential benefits against 'highly complex' malignancies. CML: chronic myeloid leukemia; APL: acute promyelocytic leukemia; HCL: hairy cell leukemia; CNL: chronic neutrophilic leukemia; AML: acute myeloid leukemia; DLBCL: diffuse large B-cell lymphoma; ALL: acute lymphoblastic leukemia; MM: multiple myeloma; T-NHL: T-cell non-Hodgkin lymphoma; OxPHOS: oxidative phosphorylation. 
cases, focal amplification of MCL1 or larger chromosomal gains at $1 \mathrm{q}$ drive venetoclax resistance. In this context, MCL-1 overexpression takes control of the anti-apoptotic dependencies at the expense of BCL-2. ${ }^{22}$ A recent work indicates that also $B A X$ variants, including missense, nonsense, frameshift, and splice site mutations, emerge in AML progressing on venetoclax. ${ }^{100}$ In AML cell lines, $B A X$ but not $B A K 1$ loss is associated with resistance to BCL-2 and MCL-1 antagonists. ${ }^{100} \mathrm{~A}$ mutation at $B A X$ c. 370 was detected in a case of mantle cell lymphoma that relapsed on venetoclax. ${ }^{101}$ In contrast to BCL2 mutations, $B A X$ alterations function as a more downstream resistance mechanism potentially affecting sensitivity to a wider range of $\mathrm{BH} 3$ mimetics, with important implications when MCL-1 and BCL-xL inhibitors become available for clinical practice. Mutations in genes that do not encode BCL-2 family members have also been implicated in resistance mechanisms. Although TP53-disrupted AML and CLL cells are sensitive to acute venetoclax treatment, they are capable of escaping chronic BCL-2 inhibition, partly because of an increased threshold for BAK/BAX activation. ${ }^{102}$ Moreover, lack of TP53 activity reduces the transcription of the pro-apoptotic genes PUMA and NOXA, potentially heightening the apoptotic threshold and impairing the efficacy of individual BH3 mimetics when used at suboptimal doses or over long periods of time. ${ }^{102}$ In AML, KRAS and PTPN11 mutations also decrease sensitivity to venetoclax. Mutant KRAS downregulates $\mathrm{BCL}-2$ and $\mathrm{BAX}$, while it upregulates MCL-1 and BCL2A1, possibly through the activation of the NF$\kappa \mathrm{B}$ pathway. Similarly, mutant PTPN11 increases the expression of BCL-xL, MCL-1 and its phosphorylated form, potentially targetable by the correspondent inhibitors. ${ }^{103}$

\section{Anti-apoptotic profiles at the forefront of integrated precision medicine}

Until very recently, the paradigm of precision medicine in clinical oncology consisted in matching the right drug with the right patient based on a tumor's genomic signature. Results from the NCI-MATCH trial indicated, however, that only $37.6 \%$ of enrolled patients had actionable alterations and only $17.8 \%$ were assigned to a treatment arm. ${ }^{104}$ Furthermore, resistance-conferring tumor mutations were found in $71.3 \%$ of specimens, thus lowering the chance of meaningful responses. ${ }^{104}$ Overall, the cooccurrence of multiple driver mutations, the poor ability to predict which mutation is a driver and which is a mere bystander, the complexity of resistance mechanisms, and the paucity of available drugs compared to the variety of genomic alterations limit the success rate of genomicdriven precision medicine. The emergence of mitochondrial apoptosis as a cancer vulnerability highlights that successful targets can be found outside genomic alterations, and that genomic alterations not always predict response to mitochondrial targeting. Indeed, BCL-2 rearrangements or expression level do not always correlate with venetoclax response. By contrast, functional assays were able to identify tumors that were more likely to respond to BCL-2 inhibition in the clinic..$^{20,49}$ Moreover, in the context of CLL, results of $\mathrm{BH} 3$ profiling correlated with lymphocyte count reduction upon venetoclax initiation in vivo. ${ }^{61}$ Although the reliability of these approaches in predicting clinical endpoints is still under investigation (e.g., NCT03943342, NCT03214562, NCT03709758), it looks clear that targeting functional cell biology domains (e.g., mitochondrial apoptosis, B-cell receptor signaling, immune interactions), in addition to the static mutational repertoire, provides therapeutic benefits in hematology. Nevertheless, there should be awareness that mitochondrial apoptosis is only one of the functional domains of cancer cell biology and, for most hematologic malignancies, mitochondrial targeting alone does not provide deep and durable remissions.

Blood cancers might be currently classified into three categories based on their underpinnings and treatment requirements. The rare 'purely genomic' malignancies have a single genetic abnormality that almost entirely sustains the neoplastic growth. In this case, genomic-driven precision medicine is a highly effective therapeutic strategy and has already gained success. CML is the prototype of these diseases that are effectively treated with molecules targeting their genetic hallmarks. ${ }^{105}$ The "mostly functional' malignancies, such as CLL, are particularly vulnerable to the targeting of selected oncogenic pathways that have been discovered through cell biology experiments rather than mutational analyses. ${ }^{106}$ While CLL cells have several mutations across their genome, the targets of the most effective drugs, such as ibrutinib and venetoclax, are never mutated..$^{106}$ In this category, knowledge built on perturbation of live cells proved perhaps more useful than DNA sequencing in terms of therapeutic applications, and targeting only one functional domain at a time yields considerable results. ${ }^{40}$ The 'highly complex' malignancies, such as AML and DLBCL, are those in which different genetic drivers frequently co-occur, and multiple functional domains simultaneously sustain cancer cell survival..$^{107}$ In this context, single-agent treatments rarely provide impressive results due to intrinsic and adaptive resistance. An integrated precision medicine approach encompassing genetic and functional testing might be needed to improve the outcome of this category, especially in the R/R setting in which tumor heterogeneity is further amplified. ${ }^{107}$ As illustrated in Figure 5, analysis on an individual basis of anti-apoptotic dependencies together with complementary static and functional measurements might help to reach this goal in the future. A prerequisite for this approach will be to design clinically applicable ex vivo assays, each with the ability to interrogate one specific domain of cancer cell biology. Because cancer cells usually maintain BAX and BAK expression, and hence are susceptible to the restoration of mitochondrial apoptosis, rational targeting of extra-mitochondrial vulnerabilities through integrated precision medicine might eventually increase apoptotic priming and enhance the effectiveness of concomitant BCL-2 family antagonism.

\section{Concluding remarks}

The clinical success of venetoclax in CLL and AML generated considerable enthusiasm on targeting apoptosis in hematology. Basic discoveries in the field will be further rewarded with the possible clinical introduction of MCL1 and BCL-xL inhibitors, which are currently under investigation. Different methods have been established to derive tumor-specific anti-apoptotic dependencies, with 
potential clinical applications. In particular, functional precision medicine platforms such as $\mathrm{BH} 3$ profiling and the BH3-mimetic toolkit have proven promising to prioritize apoptosis-targeting agents in a clinically appropriate timeframe. In several studies, they predicted clinical results more accurately than the expression of the BCL-2 family proteins or other static measurements. Moreover, these approaches are somehow breaking the paradigm of precision medicine as an omics-based concept, and pave the way for novel companion diagnostic assays based on ex vivo perturbation of live cells. To pursue this path, clinical and technical efforts will be needed to obtain adequate amounts of live cancer cells from patients, and to standardize ex vivo protocols aiming at minimizing interlaboratory variability. Despite the undoubted advantages that $\mathrm{BH} 3$ domain-related pharmacology has been provid- ing for some hematologic malignancies, it is clear that a multitude of other genetic and functional dependencies exists in complex cancers. In such cases, different angles of cell biology need to be explored simultaneously to instruct more effective combination strategies.

\section{Disclosures}

No conflicts of interest to disclose.

\section{Contributions}

$I F, A R$, and $C V$ conceived and wrote the manuscript, and reviewed the literature.

\section{Acknowledgments}

This research received no external funding. The figures were created with Biorender.com.

\section{References}

1. Carneiro BA, El-Deiry WS. Targeting apoptosis in cancer therapy. Nat Rev Clin Oncol. 2020;17(7):395-417

2. Galluzzi L, Vitale I, Aaronson SA, et al. Molecular mechanisms of cell death: recommendations of the Nomenclature Committee on Cell Death 2018. Cell Death Differ. 2018;25(3):486-541.

3. Tummers B, Green DR. Caspase-8: regulating life and death. Immunol Rev. 2017;277(1):76-89

4. Kale J, Osterlund EJ, Andrews DW. BCL-2 family proteins: changing partners in the dance towards death. Cell Death Differ. 2018;25(1):65-80.

5. Hamouda MA, Jacquel A, Robert G, et al. BCL-B (BCL2L10) is overexpressed in patients suffering from multiple myeloma (MM) and drives an MM-like disease in transgenic mice. J Exp Med. 2016;213(9): 1705-1722.

6. Letai A, Bassik MC, Walensky LD, Sorcinelli $\mathrm{MD}$, Weiler S, Korsmeyer SJ. Distinct BH3 domains either sensitize or activate mitochondrial apoptosis, serving as prototype cancer therapeutics. Cancer Cell. 2002;2(3): 183-192.

7. Moldoveanu T, Czabotar PE. BAX, BAK, and BOK: a coming of age for the BCL-2 family effector proteins. Cold Spring Harb Perspect Biol. 2020;12(4):a036319.

8. Kim H, Tu HC, Ren D, et al. Stepwise activation of BAX and BAK by tBID, BIM, and PUMA initiates mitochondrial apoptosis. Mol Cell. 2009;36(3):487-499.

9. Devan J, Janikova A, Mraz M. New concepts in follicular lymphoma biology: from BCL2 to epigenetic regulators and non-coding RNAs. Semin Oncol. 2018;45(5-6):291-302.

10. Riedell PA, Smith SM. Double hit and double expressors in lymphoma: definition and treatment. Cancer. 2018;124(24):4622-4632.

11. Pekarsky Y, Balatti V, Croce CM. BCL2 and miR-15/16: from gene discovery to treatment. Cell Death Differ. 2018;25(1):21-26.

12. Slomp A, Moesbergen LM, Gong JN, et al. Multiple myeloma with 1q21 amplification is highly sensitive to MCL-1 targeting. Blood Adv. 2019;3(24):4202-4214.

13. Liu T, Lam V, Thieme E, et al. Pharmacologic targeting of Mcl-1 induces mitochondrial dysfunction and apoptosis in B-cell lymphoma cells in a TP53- and BAX-dependent manner. Clin Cancer Res. 2021;27(17):49104922.
14. Bachmann PS, Piazza RG, Janes ME, et al Epigenetic silencing of BIM in glucocorticoid poor-responsive pediatric acute lymphoblastic leukemia, and its reversal by histone deacetylase inhibition. Blood. 2010;116(16): 3013-3022.

15. Wang JD, Katz SG, Morgan EA, Yang DT, Pan X, Xu ML. Proapoptotic protein BIM as a novel prognostic marker in mantle cell lymphoma. Hum Pathol. 2019;93:54-64.

16. Ni Chonghaile T, Sarosiek KA, Vo TT, et al. Pretreatment mitochondrial priming correlates with clinical response to cytotoxic chemotherapy. Science. 2011;334(6059): 1129-1133.

17. Cerella C, Dicato M, Diederich M. BH3 mimetics in AML therapy: death and beyond? Trends Pharmacol Sci. 2020;41(11): 793-814.

18. Del Gaizo Moore V, Brown JR, Certo M, Love TM, Novina CD, Letai A. Chronic lymphocytic leukemia requires BCL2 to sequester prodeath BIM, explaining sensitivity to BCL2 antagonist ABT-737. J Clin Invest. 2007;117(1):112-121.

19. de Jong MRW, Langendonk M, Reitsma B, et al. Heterogeneous pattern of dependence on anti-apoptotic BCL-2 family proteins upon CHOP treatment in diffuse large B-cell lymphoma. Int J Mol Sci. 2019;20(23):6036.

20. Touzeau C, Ryan J, Guerriero J, et al. BH3 profiling identifies heterogeneous dependency on Bcl-2 family members in multiple myeloma and predicts sensitivity to $\mathrm{BH} 3$ mimetics. Leukemia. 2016;30(3):761-764.

21. Grabow S, Delbridge AR, Aubrey BJ, Vandenberg CJ, Strasser A. Loss of a single Mcl-1 allele inhibits MYC-driven lymphomagenesis by sensitizing pro-B cells to apoptosis. Cell Rep. 2016;14(10):2337-2347.

22. Adams CM, Kim AS, Mitra R, Choi JK, Gong JZ, Eischen CM. BCL-W has a fundamental role in B cell survival and lymphomagenesis. J Clin Invest. 2017;127(2):635-650.

23. Dengler MA, Teh CE, Thijssen $\mathrm{R}$, et al. Potent efficacy of MCL-1 inhibitor-based therapies in preclinical models of mantle cell lymphoma. Oncogene. 2020;39(9):20092023.

24. Kelly GL, Grabow S, Glaser SP, et al. Targeting of MCL-1 kills MYC-driven mouse and human lymphomas even when they bear mutations in p53. Genes Dev. 2014;28(1):58-70.

25. Bhatt S, Pioso MS, Olesinski EA, et al. Reduced mitochondrial apoptotic priming drives resistance to $\mathrm{BH} 3$ mimetics in acute myeloid leukemia. Cancer Cell. 2020;38(6):
872-890.

26. Smith VM, Dietz A, Henz K, et al. Specific interactions of BCL-2 family proteins mediate sensitivity to $\mathrm{BH}$-mimetics in diffuse large B-cell lymphoma. Haematologica. 2020;105(8):2150-2163.

27. Ewald L, Dittmann J, Vogler M, Fulda S. Side-by-side comparison of BH3-mimetics identifies MCL-1 as a key therapeutic target in AML. Cell Death Dis. 2019;10(12):917.

28. Diepstraten ST, Chang C, Tai L, et al. BCL$\mathrm{W}$ is dispensable for the sustained survival of select Burkitt lymphoma and diffuse large B-cell lymphoma cell lines. Blood Adv. 2020;4(2):356-366.

29. Ryan J, Montero J, Rocco J, Letai A. iBH3: simple, fixable $\mathrm{BH} 3$ profiling to determine apoptotic priming in primary tissue by flow cytometry. Biol Chem. 2016;397(7):671-678.

30. Gomez-Bougie P, Maiga S, Tessoulin B, et al $\mathrm{BH} 3-$ mimetic toolkit guides the respective use of BCL2 and MCL1 BH3-mimetics in myeloma treatment. Blood. 2018;132(25): 2656-2669.

31. Samson DJ, Seidenfeld J, Ziegler K, Aronson $\mathrm{N}$. Chemotherapy sensitivity and resistance assays: a systematic review. J Clin Oncol. 2004;22(17):3618-3630.

32. Shah N, Oseth L, Tran H, Hirsch B, LeBien TW. Clonal variation in the B-lineage acute lymphoblastic leukemia response to multiple cytokines and bone marrow stromal cells. Cancer Res. 2001;61(13):5268-5274.

33. Pollyea DA, Stevens BM, Jones CL, et al. Venetoclax with azacitidine disrupts energy metabolism and targets leukemia stem cells in patients with acute myeloid leukemia. Nat Med. 2018;24(12):1859-1866

34. Del Gaizo Moore V, Letai A. BH3 profiling-measuring integrated function of the mitochondrial apoptotic pathway to predict cell fate decisions. Cancer Lett. 2013;332(2):202 205.

35. Ghavami S, Hashemi M, Ande SR, et al Apoptosis and cancer: mutations within caspase genes. J Med Genet. 2009;46(8):497510.

36. Montero J, Sarosiek KA, DeAngelo JD, et al. Drug-induced death signaling strategy rapidly predicts cancer response to chemotherapy. Cell. 2015;160(5):977-989.

37. Montero J, Letai A. Dynamic BH3 profilingpoking cancer cells with a stick. Mol Cell Oncol. 2016;3(3):e1040144.

38. Deng J, Isik E, Fernandes SM, Brown JR, Letai A, Davids MS. Bruton's tyrosine kinase inhibition increases BCL-2 dependence and enhances sensitivity to venetoclax in chronic 
lymphocytic leukemia. Leukemia. 2017;31 (10):2075-2084

39. Herbaux C, Kornauth C, Poulain S, et al. $\mathrm{BH} 3$ profiling identifies ruxolitinib as a promising partner for venetoclax to treat $\mathrm{T}$ cell prolymphocytic leukemia. Blood. 2021;137(25):3495-3506

40. Roberts AW, Davids MS, Pagel JM, et al. Targeting BCL2 with venetoclax in relapsed chronic lymphocytic leukemia. N Engl J Med. 2016;374(4):311-322

41. Konopleva M, Pollyea DA, Potluri J, et al. Efficacy and biological correlates of response in a phase II study of venetoclax monotherapy in patients with acute myelogenous leukemia. Cancer Discov. 2016;6(10):11061117.

42. Roberts AW, Wei AH, Huang DCS. BCL2 and MCL1 inhibitors for hematologic malignancies. Blood. 2021;138(13):1120-1136.

43. Pan R, Hogdal LJ, Benito JM, et al. Selective BCL-2 inhibition by ABT-199 causes on-target cell death in acute myeloid leukemia. Cancer Discov. 2014;4(3):362-375.

44. Jilg S, Reidel V, Müller-Thomas C, et al. Blockade of BCL-2 proteins efficiently induces apoptosis in progenitor cells of highrisk myelodysplastic syndromes patients. Leukemia. 2016;30(1):112-123.

45. Kadia TM, Reville PK, Borthakur G, et al. Venetoclax plus intensive chemotherapy with cladribine, idarubicin, and cytarabine in patients with newly diagnosed acute myeloid leukaemia or high-risk myelodysplastic syndrome: a cohort from a singlecentre, single-arm, phase 2 trial. Lancet Haematol. 2021;8(8):e552-e561.

46. DiNardo CD, Pratz K, Pullarkat V, et al. Venetoclax combined with decitabine or azacitidine in treatment-naive, elderly patients with acute myeloid leukemia. Blood. 2019;133(1):7-17.

47. Pei S, Pollyea DA, Gustafson A, et al. Monocytic subclones confer resistance to venetoclax-based therapy in patients with acute myeloid leukemia. Cancer Discov. 2020;10(4):536-551

48. Del Gaizo Moore V, Schlis KD, Sallan SE, Armstrong SA, Letai A. BCL-2 dependence and ABT-737 sensitivity in acute lymphoblastic leukemia. Blood. 2008;111(4): 2300-2309.

49. Seyfried F, Demir S, Horl RL, et al. Prediction of venetoclax activity in precursor B-ALL by functional assessment of apoptosis signaling. Cell Death Dis. 2019;10(8):571

50. Alford SE, Kothari A, Loeff FC, et al. BH3 inhibitor sensitivity and Bcl-2 dependence in primary acute lymphoblastic leukemia cells. Cancer Res. 2015;75(7):1366-1375

51. Khaw SL, Suryani S, Evans K, et al. Venetoclax responses of pediatric ALL xenografts reveal sensitivity of MLLrearranged leukemia. Blood. 2016;128(10): 1382-1395

52. Pullarkat VA, Lacayo NJ, Jabbour E, et al. Venetoclax and navitoclax in combination with chemotherapy in patients with relapsed or refractory acute lymphoblastic leukemia and lymphoblastic lymphoma. Cancer Discov. 2021;11(6):1440-1453

53. Benito JM, Godfrey L, Kojima K, et al. MLLrearranged acute lymphoblastic leukemias activate BCL-2 through H3K79 methylation and are sensitive to the BCL-2-specific antagonist ABT-199. Cell Rep. 2015;13(12): 2715-2727.

54. Chonghaile TN, Roderick JE, Glenfield C, et al. Maturation stage of T-cell acute lymphoblastic leukemia determines BCL-2 versus BCL-XL dependence and sensitivity to
ABT-199. Cancer Discov, 2014:4(9):1074 1087

55. Di Grande A, Peirs S, Donovan PD, et al. The spleen as a sanctuary site for residual leukemic cells following ABT-199 monotherapy in ETP-ALL. Blood Adv. 2021;5(7):1963-1976

56. Moia R, Patriarca A, Schipani M, Gaidano G. The biology of chronic lymphocytic leukemia: diagnostic and prognostic implications. Cancer J. 2021;27(4):266-274

57. Gohil SH, Wu CJ. Dissecting CLL through high-dimensional single-cell technologies. Blood. 2019;133(13):1446-1456.

58. Mavridou D, Psatha K, Aivaliotis M. Proteomics and drug repurposing in CLI towards precision medicine. Cancers (Basel) 2021;13(14):3391.

59. Hanada M, Delia D, Aiello A, Stadtmauer E, Reed JC. Bcl-2 gene hypomethylation and high-level expression in B-cell chronic lymphocytic leukemia. Blood. 1993;82(6):1820 1828

60. Fang H, Reichard KK, Rabe KG, et al. IGH translocations in chronic lymphocytic leukemia: clinicopathologic features and clinical outcomes. Am J Hematol. 2019:94(3):338-345.

61. Anderson MA, Deng J, Seymour JF, et al. The BCL2 selective inhibitor venetoclax induces rapid onset apoptosis of CLL cells in patients via a TP53-independent mechanism. Blood. 2016;127(25):3215-3224.

62. Davids MS, Deng J, Wiestner A, et al. Decreased mitochondrial apoptotic priming underlies stroma-mediated treatment resistance in chronic lymphocytic leukemia. Blood. 2012;120(17):3501-3509.

63. Hillmen P, Rawstron AC, Brock K, et al. Ibrutinib plus venetoclax in relapsed/refractory chronic lymphocytic leukemia: the CLARITY study. J Clin Oncol. 2019;37(30): 2722-2729.

64. Morin RD, Arthur SE, Hodson DJ. Molecular profiling in diffuse large B-cell lymphoma: why so many types of subtypes? Br J Haematol. 2022;196(4):814 829.

65. Rys RN, Wever CM, Geoffrion D, et al. Apoptotic blocks in primary non-Hodgkin $B$ cell lymphomas identified by $\mathrm{BH} 3$ profiling. Cancers (Basel). 2021:13(5):1002.

66. Wenzel SS, Grau M, Mavis C, et al. MCL1 is deregulated in subgroups of diffuse large $\mathrm{B}-$ cell lymphoma. Leukemia. 2013·27(6):13811390

67. Klanova M, Klener P. BCL-2 Proteins in pathogenesis and therapy of B-cell nonHodgkin lymphomas. Cancers (Basel). 2020;12(4):938.

68. Bojarczuk K, Wienand K, Ryan JA, et al. Targeted inhibition of PI3Kalpha/delta is synergistic with BCL-2 blockade in genetically defined subtypes of DLBCL. Blood. 2019;133(1):70-80

69. Davids MS, Roberts AW, Seymour JF, et al Phase I first-in-human study of venetoclax in patients with relapsed or refractory nonHodgkin lymphoma. I Clin Oncol. 2017:35(8):826-833

70. Davids MS, Roberts AW, Kenkre VP, et al. Long-term follow-up of patients with relapsed or refractory non-Hodgkin lymphoma treated with venetoclax in a phase I, first-in-human study. Clin Cancer Res. 2021:27(17):4690-4695.

71. Serrat N, Guerrero-Hernandez M, MatasCespedes A, et al. PI3Kdelta inhibition reshapes follicular lymphoma-immune microenvironment cross talk and unleashes the activity of venetoclax. Blood Adv.
2020:4(17):4217-4231

72. Punnoose EA, Leverson JD, Peale F, et al Expression profile of BCL-2, BCL-XL, and MCL-1 predicts pharmacological response to the BCL-2 selective antagonist venetoclax in multiple myeloma models. Mol Cancer Ther. 2016;15(5):1132-1144.

73. Hanamura I, Stewart JP, Huang Y, et al Frequent gain of chromosome band 1q21 in plasma-cell dyscrasias detected by fluorescence in situ hybridization: incidence increases from MGUS to relapsed myeloma and is related to prognosis and disease progression following tandem stem-cell transplantation. Blood. 2006;108(5):1724-1732

74. Wuilleme-Toumi S, Robillard N, Gomez P, et al. Mcl-1 is overexpressed in multiple myeloma and associated with relapse and shorter survival. Leukemia. 2005;19(7):12481252

75. Gupta VA, Matulis SM, Conage-Pough JE, et al. Bone marrow microenvironment-derived signals induce Mcl-1 dependence in multiple myeloma. Blood. 2017;129(14):1969-1979.

76. Wang M, Wu D, Liu P, Deng J. Silence of MCL-1 upstream signaling by shRNA abrogates multiple myeloma growth. Exp Hematol Oncol. 2014;3(1):27.

77. Algarin EM, Diaz-Tejedor A, Mogollon P, et al. Preclinical evaluation of the simultaneous inhibition of MCL-1 and BCL-2 with the combination of $\mathrm{S} 63845$ and venetoclax in multiple myeloma. Haematologica. 2020; 105(3):e116-e120

78. Touzeau C, Maciag P, Amiot M, Moreau P. Targeting Bcl-2 for the treatment of multiple myeloma. Leukemia. 2018;32(9):1899-1907.

79. Kumar S, Kaufman JL, Gasparetto C, et al. Efficacy of venetoclax as targeted therapy for relapsed/refractory $t(11 ; 14)$ multiple myeloma. Blood. 2017;130(22):2401-2409.

80. Koch R, Christie AL, Crombie JL, et al. Biomarker-driven strategy for MCL1 inhibition in T-cell lymphomas. Blood. 2019;133 (6):566-575

81. Spinner S, Crispatzu G, Yi JH, et al. Re-activation of mitochondrial apoptosis inhibit T-cell lymphoma survival and treatment resistance. Leukemia. 2016:30(7):1520-1530.

82. He Y, Koch R, Budamagunta V, et al DT2216-a Bcl-xL-specific degrader is highly active against $\mathrm{Bcl}$-xL-dependent $\mathrm{T}$ cell lymphomas. J Hematol Oncol. 2020;13(1):95.

83. Smith VM, Lomas O, Constantine D, et al. Dual dependence on BCL2 and MCL1 in T cell prolymphocytic leukemia. Blood Adv. 2020;4(3):525-529.

84. Kornauth C, Herbaux C, Boidol B, et al. Rationale for the combination of venetoclax and ibrutinib in T-prolymphocytic leukemia Haematologica. 2021;106(8):2251-2256.

85. Adams CM, Mitra R, Vogel AN, Liu J, Gong IZ, Eischen CM. Targeting BCL-W and BCLXI as a therapeutic strateoy for Hodgkin lymphoma. Leukemia. 2020;34(3):947-952.

86. Montero J, Stephansky J, Cai T, et al. Blastic plasmacytoid dendritic cell neoplasm is dependent on BCL2 and sensitive to venetoclax. Cancer Discov. 2017;7(2):156-164.

87. Beziat G, Ysebaert L, Gaudin C, Steinmeyer Z, Balardy L. Venetoclax to treat relapsed blastic plasmacytoid dendritic cell neoplasm: a case-report and review of literature. Leuk Res. 2019;85:106199.

88. Agha ME, Monaghan SA, Swerdlow SH. Venetoclax in a patient with a blastic plasmacytoid dendritic-cell neoplasm. $\mathrm{N}$ Engl J Med. 2018;379(15):1479-1481.

89. Blombery P, Thompson ER, Nguyen T, et al. Multiple BCL2 mutations cooccurring with Gly101Val emerge in chronic lymphocytic 
leukemia progression on venetoclax. Blood. 2020;135(10):773-777.

90. Yu X, Munoz-Sagredo L, Streule K, et al. CD44 loss of function sensitizes AML cells to the BCL-2 inhibitor venetoclax by decreasing CXCL12-driven survival cues. Blood. 2021;138(12):1067-1080.

91. Haselager M, Thijssen R, West C, et al. Regulation of Bcl-XL by non-canonical NFkappaB in the context of CD40-induced drug resistance in CLL. Cell Death Differ. 2021;28(5):1658-1668.

92. Guieze R, Liu VM, Rosebrock D, et al. Mitochondrial reprogramming underlies resistance to BCL-2 inhibition in lymphoid malignancies. Cancer Cell. 2019;36(4):369384.

93. Bixby D, Talpaz M. Mechanisms of resistance to tyrosine kinase inhibitors in chronic myeloid leukemia and recent therapeutic strategies to overcome resistance. Hematology Am Soc Hematol Educ Program. 2009;461-476.

94. Haselager MV, Kielbassa K, Ter Burg J, et al. Changes in Bcl-2 members after ibrutinib or venetoclax uncover functional hierarchy in determining resistance to venetoclax in CLL. Blood. 2020;136(25):2918-2926.

95. Stevens BM, Jones CL, Pollyea DA, et al.
Fatty acid metabolism underlies venetoclax resistance in acute myeloid leukemia stem cells. Nat Cancer. 2020;1(12):1176-1187.

96. Roca-Portoles A, Rodriguez-Blanco G Sumpton D, et al. Venetoclax causes metabolic reprogramming independent of BCL-2 inhibition. Cell Death Dis. 2020;11(8):616.

97. Jones CL, Stevens BM, Pollyea DA, et al. Nicotinamide metabolism mediates resistance to venetoclax in relapsed acute myeloid leukemia stem cells. Cell Stem Cell. 2020;27(5):748-764

98. Chen X, Glytsou C, Zhou H, et al. Targeting mitochondrial structure sensitizes acute myeloid leukemia to venetoclax treatment. Cancer Discov. 2019;9(7):890-909.

99. Sharon D, Cathelin S, Mirali S, et al. Inhibition of mitochondrial translation overcomes venetoclax resistance in AML through activation of the integrated stress response. Sci Transl Med. 2019;11(516): eaax2863.

100. Moujalled DM, Brown FC, Pomilio G, et al. Acquired mutations in BAX confer resistance to $\mathrm{BH} 3$ mimetics in acute myeloid leukemia. Blood. 2020;136(Suppl 1):7-8.

101. Thompson ER, Nguyen T, Kankanige Y, et al. High clonal complexity of resistance mechanisms occurring at progression after single-agent targeted therapy strategies in chronic lymphocytic leukemia. Blood. 2020;136(Suppl 1):15-16.

102. Thijssen R, Diepstraten ST, Moujalled D, et al. Intact TP-53 function is essential for sustaining durable responses to $\mathrm{BH} 3$-mimetic drugs in leukemias. Blood. 2021;137(20): 2721-2735.

103. Zhang H, Nakauchi Y, Kohnke T, et al Integrated analysis of patient samples identifies biomarkers for venetoclax efficacy and combination strategies in acute myeloid leukemia. Nat Cancer. 2020;1(8):826-839.

104. Flaherty KT, Gray RJ, Chen AP, et al Molecular landscape and actionable alterations in a genomically guided cancer clinical trial: National Cancer Institute Molecular Analysis for Therapy Choice (NCIMATCH). J Clin Oncol. 2020;38(33):38833894

105. Hehlmann R. Chronic myeloid leukemia in 2020. Hemasphere. 2020;4(5):e468.

106. Letai A. Functional precision cancer medicine-moving beyond pure genomics. Nat Med. 2017;23(9):1028-1035

107. Adashek JJ, Subbiah V, Kurzrock R. From tissue-agnostic to N-of-one therapies: (r)evolution of the precision paradigm. Trends Cancer. 2021;7(1):15-28. 